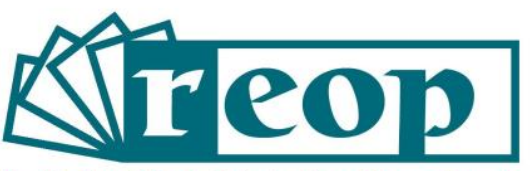

Revista Española de Orientación y Psicopedagogía

\title{
MEJORA DE LAS PRÁCTICAS ORIENTADORAS DESDE LA REVISIÓN SISTEMÁTICA DE ESTUDIOS SOBRE EFICACIA ESCOLAR ${ }^{1}$
}

\author{
IMPROVEMENT OF GUIDANCE PRACTICES: A SYSTEMATIC REVIEW OF \\ STUDIES ON SCHOOL EFFECTIVENESS
}

\author{
Pablo Delgado-Galindo \\ Universidad de Sevilla. Facultad Ciencias de la Educación. Departamento Métodos de \\ Investigación y Diagnóstico en Educación. Sevilla, España \\ Javier Rodríguez-Santero \\ Universidad de Sevilla. Facultad Ciencias de la Educación. Departamento Métodos de \\ Investigación y Diagnóstico en Educación. Sevilla, España \\ Juan-Jesús Torres-Gordillo \\ Universidad de Sevilla. Facultad Ciencias de la Educación. Departamento Métodos de \\ Investigación y Diagnóstico en Educación. Sevilla, España
}

\section{RESUMEN}

Para contribuir al desarrollo integral del alumnado, principio básico de la orientación educativa, es necesario conocer las variables contextuales que afectan a la eficacia escolar (EE), entendida esta en términos de rendimiento académico. Es necesario estudiar el conjunto de factores que explica por qué un centro educativo posee resultados distintos a los que cabría esperar, atendiendo a su contexto social, económico y cultural. El informe Coleman (1966), que podría

\footnotetext{
1 Fuente de financiación: Este proyecto fue cofinanciado por el Fondo Europeo de Desarrollo Regional (FEDER) y la Consejería de Economía, Conocimiento, Empresas y Universidad de la Junta de Andalucía, titulado "Diagnóstico del rendimiento educativo en educación primaria: un primer paso hacia la eficacia escolar", con código US-1263333. Fue obtenido en una convocatoria competitiva de proyectos I+D+i, de la Secretaría General de Universidades, Investigación y Tecnología, del programa operacional FEDER 2014-2020, convocatoria 2018.

2 Correspondencia: Pablo Delgado-Galindo. Correo-e: pdgalindo@us.es
} 
considerarse el precursor de este tipo de estudios, concluyó que el contexto del que procede el alumnado es lo más determinante en su progreso educativo. Numerosos investigadores han intentado rebatir esta conclusión, siendo uno de los tópicos de mayor actualidad en la orientación educativa. El objetivo de este artículo es aportar información sobre cuáles son las variables contextuales que guardan mayor relación con la EE y, a partir de ellas, contribuir a la mejora de la orientación educativa. Para tal fin, se ha desarrollado una revisión sistemática que ha supuesto la lectura en profundidad y el análisis cruzado de 64 documentos que recogen investigaciones en las que se abordan variables contextuales que afectan al rendimiento educativo del alumnado. Como resultado de este estudio se ha logrado conocer cuáles son las variables contextuales, coadyuvantes en la explicación de la EE, que pueden ayudarnos a contextualizar la orientación educativa. Las conclusiones alcanzadas revelan cómo podrían abordarse, desde la orientación educativa, estas variables que tan determinantemente marcan el devenir del alumnado en el centro educativo.

Palabras clave: orientación pedagógica, tutoría, eficiencia de la educación, rendimiento escolar, ambiente educacional.

\section{ABSTRACT}

Contributing to the holistic development of students is a basic principle of educational guidance, and it requires knowledge of the contextual variables that affect school effectiveness (SE), understood in terms of academic performance. It is necessary to study the set of factors that explain why an educational center has different results than expected based on its social, economic, and cultural context. The Coleman report (1966), which could be considered the precursor of this type of study, concluded that the context students come from is the determining factor in their educational progress. Numerous researchers have tried to refute this conclusion, which is one of the most current topics in educational guidance. The aim of this article is to provide information about which contextual variables are the most related to SE, and, in order to contribute to the improvement of educational guidance. To this end, a systematic review has been carried out involving in-depth reading and cross-analysis of 64 documents, that gather research addressing the contextual variables that affect students' educational performance. As a result of this study, it has been possible to find out which are the contextual variables that contribute to the explanation of SE, allowing us to contextualise educational guidance. The conclusions reached reveal how these variables, which so decisively mark students' development in school, could be addressed through educational guidance.

Key Words: school effectiveness, academic achievement, school guidance, tutorial programs, learning environment.

\section{Cómo citar este artículo:}

Delgado-Galindo, P., Rodríguez-Santero, J. y Torres-Gordillo, J.-J. (2021). Mejora de las prácticas orientadoras desde la revisión sistemática de estudios sobre eficacia escolar. Revista Española de
Orientación
$y$
Psicopedagogía,
$32(3)$,
93-111.

https://doi.org/10.5944/reop.vol.32.num.3.2021.32557 


\section{Introducción}

La orientación es un componente clave de la educación que favorece el desarrollo integral del alumnado (Ibáñez-Martínez, 2020; Martínez-Clares et al., 2016). Atendiendo a su principio de intervención social, la orientación engloba todas las vertientes del desarrollo humano, incluyendo su contexto social. Los tutores y orientadores necesitan implicarse, como agentes de cambio social, en el estudio de las variables contextuales que afectan a la eficacia escolar (EE) (ÁlvarezGonzález y Bisquerra, 2012; Ramírez-García et al., 2020).

Tomando como referencia a Núñez (2007), entendemos las variables contextuales como las características del ambiente cultural, social, escolar y personal de los estudiantes. La comprensión de estas variables implica poder abordar la orientación educativa desde la visión que actualmente se propone. Es decir, desde un modelo de orientación basado en el desarrollo integral del alumnado, que tenga en cuenta aspectos académicos, personales, profesionales, sociales y que contribuya a incrementar la calidad de la educación (Amor-Almedina y Serrano-Rodríguez, 2019).

La eficacia escolar (EE) se asocia al rendimiento educativo del alumnado y se interesa por el estudio de aquellos cambios, planificados por los centros educativos, que producen mejoras en los resultados académicos de los escolares (Hopkins et al., 1994). Murillo (2005) establece que un centro posee EE cuando el rendimiento educativo de su alumnado es mayor de lo que cabría esperar (residuo positivo), teniendo en cuenta los factores socioeconómicos y culturales de los estudiantes y sus rendimientos previos. Para Blanco (2007), un centro podrá considerarse eficaz únicamente si los residuos positivos se mantienen a lo largo del tiempo.

El Informe Coleman (Coleman et al., 1966), considerado el precursor de los estudios sobre EE, concluyó que la educación recibida por el alumnado en las escuelas incidía muy poco en su desarrollo, debido a que el contexto socioeconómico y cultural del que procedían era lo más determinante en su progreso educativo. Numerosas investigaciones han intentado rebatir esta conclusión tan pesimista para la educación, dando origen al denominado movimiento de Mejora de la Escuela (School Improvement) que defiende que la educación se debe cambiar desde el centro educativo (Hargreaves, 1986). Weber (1971) expone diversas variables del proceso educativo, como el clima escolar o el liderazgo, que también influyen en el nivel de aprendizaje del alumnado. Edmonds (1979) presenta una serie de características que diferencian a los centros de alta y baja eficacia escolar, al margen del contexto socioeconómico al que pertenecen. Brookover et al. (1979), Rutter et al. (1979) y Mortimore et al. (1988), convencidos de la importancia de las variables educativas, realizaron estudios con grandes muestras de alumnos, recogiendo de forma rigurosa información sobre los procesos de enseñanza-aprendizaje desarrollados en las aulas. Estos autores consolidaron lo que pasó a denominarse investigaciones entrada-proceso-producto. Muchos estudios posteriores (Brandsma, 1993; Hill et al., 1995; Sammoms et al., 1997), basándose en ese modelo de investigación, introdujeron técnicas cualitativas y efectuaron observaciones minuciosas en el interior de las aulas, dando lugar a que el modelo evolucionara hacia lo que se conoce como contexto-entrada-proceso-producto. Posteriormente, y como consecuencia de este, surge la línea de investigación denominada "Mejora de la Eficacia Escolar" (Effectiveness School Improvement), que aúna los movimientos de Eficacia Escolar y Mejora de la Escuela (Murillo, 2003), estableciendo las bases de lo que hoy es un campo consolidado de investigación que, paulatinamente, está contribuyendo a la mejora de la calidad y la equidad en la educación. 
Las investigaciones recientes (Alm et al., 2019; Costa y Araújo, 2018; Brault et al., 2014; Huang y Shih, 2017; Ramberg et al., 2019; Saminathen et al., 2018) están centradas en descubrir cuáles son los factores, a nivel de centro y aula, que llevan a determinados centros educativos a lograr niveles altos de rendimiento y equidad entre su alumnado, pese a la situación adversa de su entorno. Esta tendencia se ha visto incrementada gracias a la relevancia que han adquirido las evaluaciones escolares de carácter externo, tales como el Programme for International Student Assessment (PISA) y el Trends in International Mathematics and Science Study (TIMSS). Estas evaluaciones presentan la limitación de no tener en cuenta particularidades asociadas con las desigualdades educativas, sociales y económicas registradas en cada centro, lo que conlleva que se generen resultados poco exactos. No es correcto asumir que el efecto de las variables contextuales sobre la EE es el mismo para los estudiantes de distintos países y centros educativos. Por otro lado, el desconocimiento del comportamiento específico de las variables contextuales en cada centro dificulta la creación de estrategias de intervención orientadora específicas para cada uno de ellos, que permita solucionar o reducir las desigualdades educativas.

Estudiar cuáles son las variables contextuales que afectan al rendimiento educativo es la base para el desarrollo de acciones orientadoras que contribuyan a la excelencia y la equidad educativa. Estudios como el de Lizasoain y Angulo (2014) ofrecen evidencias de que en los centros educativos las variables contextuales siguen una estructura anidada y jerarquizada, de manera que existen variables relevantes al nivel del estudiante y otras al nivel de aula o de centro. Por esta razón, las propuestas de actuación orientadora y acción tutorial a llevar a cabo en el centro educativo deben basarse en evaluaciones del rendimiento que hayan tomado en consideración las variables contextuales que afectan al centro y a su alumnado (Weinstein y Hernández, 2016; Wolhuter et al., 2016), procurando un desarrollo integral del individuo (OlmosGómez et al., 2019). Atendiendo a esta necesidad, la finalidad del trabajo presentado es aportar evidencias sobre las variables contextuales a nivel de entorno, centro, aula y alumno que inciden en la EE, con objeto de aportar las bases para el desarrollo de propuestas adaptadas de orientación educativa.

\section{Método}

Se ha recurrido a la estrategia metodológica de la revisión sistemática (RS), también denominada análisis documental por autores como Bisquerra (2012). Esta implica, generalmente, un proceso inductivo que tiene por objeto recabar toda evidencia empírica que cumpla los criterios de elegibilidad previamente establecidos y contribuya a responder la pregunta de investigación planteada. Implica el uso de métodos sistemáticos y explícitos para minimizar sesgos y proporcionar resultados fiables, a partir de los que extraer conclusiones que orienten la toma de decisiones (Aguilera-Eguía, 2014; Ferreira-González et al., 2011; Higgins y Green, 2011).

En el presente estudio, la pregunta de investigación es: ¿cuáles son las variables contextuales que afectan a la EE y pueden contribuir a mejorar la orientación educativa? Partiendo de este interrogante y tomando como referencia los criterios establecidos en las investigaciones de Tacconelli (2010) y Urrútia y Bonfill (2010), descritos por Prendes-Espinosa et al. (2020), se han sometido a revisión las publicaciones entre 2014 y 2019 que abordaban las variables contextuales en la explicación de la EE. Concretamente, se han analizado 24 artículos de revistas indexadas en Journal Citation Reports (JCR), 24 artículos en Scimago Journal Rank (SJR) y 8 en Emerging Sources Citation Index (ESCl), 2 libros y 1 capítulo de libro publicados en editoriales presentes en 
Scholarly Publishers Indicators (SPI) y 5 tesis doctorales ubicadas en repositorios institucionales de prestigio. Posteriormente, se han sintetizado todos los datos obtenidos para redactar los resultados de forma agrupada, clara y concisa.

Los pasos seguidos en el proceso inductivo que implica la RS desarrollada atienden a los establecidos en las investigaciones de Higgins y Green (2011) y Sobrido y Rumbo-Prieto (2018):

- Búsqueda sistemática y exhaustiva de investigaciones en Web of Science y ERIC. Descriptores empleados: school effectiveness, learning results, effectiveness, effective teaching, educational effectiveness, student achievement. Se emplearon los términos de búsqueda comunes en el ámbito de investigación en el que se enmarca el tópico de interés, con objeto de acceder al mayor número posible de resultados. No obstante, en la Web of Science hubo que incluir el filtro Social Sciences para acotar la búsqueda y obtener resultados más precisos.

- Clasificación de estudios. Se desestimaron todas aquellas publicaciones que no mostraban el efecto de alguna variable contextual en la explicación de la EE. La lectura detenida del resumen de las obras permitió seleccionar aquellas que inicialmente parecían adecuadas para responder a la pregunta de la investigación.

- Selección de investigaciones adecuadas para los propósitos de la investigación. De las más de cien publicaciones consideradas tras la primera criba, en esta fase se seleccionaron únicamente aquellas que explicitaban, de forma concreta, la relevancia de alguna variable contextual en la explicación del rendimiento académico. El contexto geográfico y el nivel educativo no se establecieron como criterios específicos de selección, de ahí que las investigaciones que finalmente se han analizado pertenezcan a diversos países del mundo y presenten resultados tanto a nivel de educación primaria, como de secundaria.

- Las publicaciones objeto de una RS deben garantizar su calidad y pertinencia en función de tres aspectos fundamentales: el rigor, la credibilidad y la relevancia. Por ello, los documentos que superaron la segunda criba fueron evaluados de acuerdo con cuatro criterios previamente establecidos: relevancia del contenido para dar respuesta a la pregunta objeto de interés en el estudio que se presenta, claridad en la formulación del objetivo de investigación, solidez del diseño metodológico empleado y rigor en el análisis de los datos. Finalmente, se seleccionaron 64 publicaciones que fueron agrupadas en función de las variables contextuales sobre las que aportaban resultados [ver Tabla 1 en https://dx.doi.org/10.17504/protocols.io.bpbnmime].

- Lectura cruzada y comparativa de las obras que abordaban una misma variable contextual, prestando especial atención a la similitud o discrepancia de los resultados obtenidos.

- Diseño del mapa visual de variables contextuales a considerar en el estudio de la EE.

\section{Resultados}

Los resultados de este estudio muestran las diferentes variables contextuales que influyen en la EE resultantes de la RS efectuada. Las variables que se exponen en la Figura 1 son las que, 
según los estudios analizados, afectan más a la EE, señalando el \% de aparición. Como puede apreciarse, el estatus socioeconómico de las familias del alumnado (44\%) resulta, con diferencia, la más estudiada en las investigaciones revisadas. Además, es la variable que más influye en el rendimiento educativo del alumnado (Abadía et al., 2018; Tourón et al., 2018; Wolhuter et al., 2016).

Figura 1.

Síntesis de las variables analizadas y porcentaje de presencie en las investigaciones examinadas

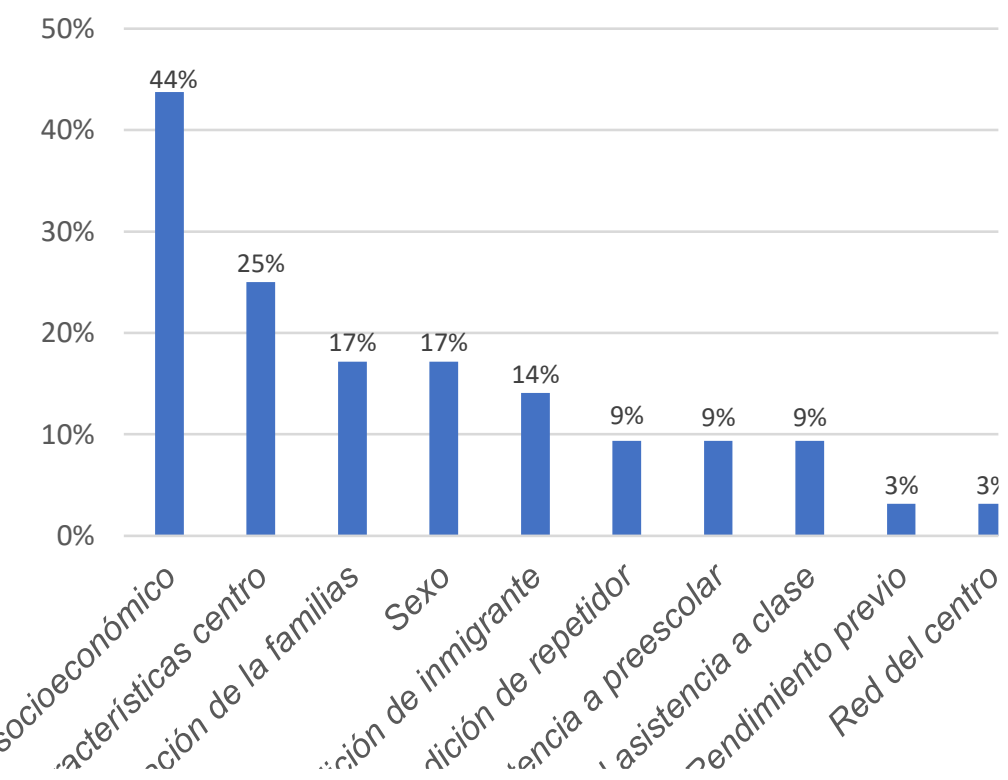

Fuente: Elaboración propia

- Índice socioeconómico y cultural de las familias (ISEC)

Numerosos estudios (Alm et al., 2019; Condliffe et al., 2015; Gamazo et al., 2018; Hanushek y Woessmann, 2017; Lizasoain y Angulo, 2014; McCardle y Hadwin, 2015; Pedroza et al., 2018; Perry, 2017; Ramberg et al., 2019; Weinstein y Hernández, 2016) han resaltado la influencia del ISEC en la EE, tanto a nivel centro como a nivel alumno.

EI ISEC influye de manera muy importante en el rendimiento (Ben-Eliyahu y Bernacki, 2015; Hendrick et al., 2016; Parra, 2018; Román-Carrasco, 2014). También afecta a la Competencia Lingüística, Matemática, Científica y Social y Ciudadana. Por cada aumento de la desviación típica del ISEC, el rendimiento de las cuatro competencias sube más de 0.25 desviaciones típicas (Martínez-Garrido y Murillo, 2016b; Murillo et al., 2015).

El alumnado de centros eficaces tiene acceso en casa a más cantidad de libros y recursos didácticos, estableciéndose una fuerte relación entre estos y el rendimiento en Lectura, con coeficientes de regresión entre 21 y 27 puntos (Costa y Araújo, 2018). La costumbre de leer libros a los hijos desde pequeños también influye positivamente sobre la EE, sobre todo en la asignatura de Lengua (Costa y Araújo, 2018; Kalb y van Ours, 2014). 
El nivel educativo de las familias tiene una incidencia muy positiva y significativa en el rendimiento del alumnado (Cordero et al., 2014; Gustafsson y Hansen, 2018; OECD, 2016; Saminathen et al., 2018). Algunas investigaciones demuestran que las madres de centros eficaces muestran mayor nivel académico que las madres de centros menos eficaces (Figueroa-Gutiérrez, 2017; Travitzki, 2017). Por el contrario, el nivel educativo del padre no parece influir significativamente en el aprendizaje de los hijos (Cordero y Manchón, 2014; Ibáñez-Martín y Formichella, 2017; Suárez-Enciso et al., 2016).

El contexto cercano del centro también influye en la EE. El rendimiento en Lectura y Matemáticas aumenta en 6 puntos por cada desviación típica que suba el nivel socioeconómico del barrio del centro (Huang y Shih, 2017; Ibáñez-Martín y Formichella, 2017; Martínez-Garrido y Murillo, 2016b).

Pese a estas evidencias, algunos autores defienden que esta relación no es tan significativa (Baş et al., 2017; Fernández-Fernández et al., 2016). Castro-Morera et al. (2014) constataron que los estudiantes no señalan los problemas económicos de sus familias como causa principal de sus bajas calificaciones. Hay que tener en cuenta que el hecho de que los estudiantes no lo perciban, no implica que esta variable no afecte al rendimiento.

Por último, Hanushek y Woessmann (2017) y Jeynes (2015) destacaron que el rendimiento educativo del alumnado en países con un alto gasto en educación no se diferencia significativamente del obtenido por el alumnado que residía en países con bajo nivel de gasto en educación. A pesar de esto, PISA 2015 sí observa una relación positiva entre el rendimiento en Ciencias y el gasto medio por alumnado en los países estudiados (OECD, 2016).

\section{- Características del centro escolar}

Si los recursos materiales del centro educativo son los apropiados y se ajustan al proceso de enseñanza-aprendizaje, influyen tanto en el desarrollo cognitivo como en el socioafectivo del alumnado (James et al., 2016; Martínez-Garrido y Murillo, 2016a; McEwan, 2015). Distintos estudios (Martínez-Garrido y Murillo, 2016c; Martínez-Garrido y Murillo, 2016d; Travitzki, 2017) señalan que disponer de biblioteca en el centro y ordenadores en las aulas, mejora el rendimiento escolar en Lengua y Matemáticas.

Los centros eficaces poseen grandes bibliotecas, niveles elevados de orden y limpieza en las aulas e infraestructuras de gran calidad (Figueroa-Gutiérrez, 2017). En ellas, los docentes están más satisfechos con los aspectos físicos del centro y sus materiales escolares, que los docentes de centros menos eficaces (Figueroa-Gutiérrez, 2017). Estos elementos predisponen al alumnado al estudio y son importantes para su bienestar (Ballesteros-Velázquez et al., 2014; Brault et al., 2014; Murillo et al., 2016; Hernández-Castilla et al., 2014).

Algunos estudios señalan que la ratio profesor-alumno posee un efecto moderado y negativo sobre la EE (Cordero y Manchón, 2014; Fernández-Fernández et al., 2016; Ibáñez-Martín y Formichella, 2017; lyer y Moore, 2017; Merlo-Martínez, 2015), mientras que otros afirman que el tamaño del centro y de las clases no tienen ninguna influencia significativa, salvo en los centros donde sí existe baja EE (Lizasoain et al., 2016; Hanushek y Woessmann, 2017).

\section{- Rendimiento previo del alumnado}

El rendimiento previo o calificaciones del curso anterior en las competencias evaluadas es un factor con elevada significatividad predictiva en las puntuaciones (Lizasoain et al., 2016). Martínez-Garrido y Murillo (2016b, d) indican que el nivel de Matemáticas y el de Lengua, aumentan 0.41 y 0.39 puntos, respectivamente, cada vez que suban un punto las calificaciones previas del alumnado. 


\section{- Condición de alumno repetidor}

La condición de repetidor, tras el ISEC familiar, es la variable que determina en mayor medida el rendimiento en Matemáticas, Lengua y Ciencias (Cordero y Manchón, 2014; Gamazo et al., 2018; Lizasoain et al., 2016; Suárez-Enciso et al., 2016). Los alumnos repetidores obtienen, por lo general, peores calificaciones en las competencias señaladas. Además, las variables que influyen en la repetición del alumnado en Educación Primaria no están relacionadas con los aspectos cognitivos del desarrollo (Cordero et al., 2014).

\section{- Condición de alumnado inmigrante}

Los alumnos que cuentan con una lengua materna diferente tienen un desarrollo escolar inferior al del alumnado nativo (Martínez-Garrido, 2015). Gamazo et al. (2018) indican que ser alumno inmigrante de primera generación, considerando a estos como las personas que han nacido fuera del país al que han emigrado (Rumbaut y Portes, 2001; Danico, 2004), tiene un efecto negativo y significativo para las asignaturas de Matemáticas y Ciencias. Ser nativo influye de forma importante y se posiciona como la variable más significativa en el rendimiento en Lengua y Lectura (Martínez et al., 2017). El alumnado inmigrante obtiene entre 4 y 5 puntos menos que los niños nativos en las Competencias Lingüística, Matemática y Científica, mientras que en la Competencia Social y Ciudadana las puntuaciones se mantienen similares (Murillo et al., 2014). Martínez-Garrido y Murillo (2016b, c) observaron que los estudiantes inmigrantes obtenían 8 puntos menos en Matemáticas. En Lengua, estos obtenían 4 puntos menos. Según PISA 2015, los alumnos inmigrantes obtienen casi la mitad de una desviación estándar por debajo del rendimiento medio en Ciencias, ya sean de primera o segunda generación (OECD, 2016).

Además, formar parte de una etnia minoritaria conlleva un desarrollo socioafectivo menor, aunque pertenecer a este grupo no demostró influencia significativa en el desarrollo cognitivo (Martínez-Garrido y Murillo, 2016d).

Otros estudios como los de Gil-Flores (2014) defienden que la relación existente entre porcentaje de alumnado inmigrante en un centro y rendimiento académico es poco significativa.

\section{- $\quad$ Red (pública, privada o concertada)}

El estudio de Hofman et al. (2015) encontró que los centros públicos obtienen peores índices de rendimiento que los concertados y estos, a su vez, son menos eficaces que los privados. Los centros públicos poseen mayor dispersión en los índices de eficacia relativa, seguidos por los concertados y, en menor medida, los privados. Por otro lado, los alumnos inmigrantes de primera generación, normalmente, acuden a centros públicos o concertados, influyendo estos en el ISEC medio del centro (Merlo-Martínez, 2015; Saminathen et al., 2018; Suárez-Enciso et al., 2016). Sin embargo, la diferencia de rendimiento entre centros públicos, privados y concertados no es lo suficientemente importante como para considerar que la red es una variable contextual relevante en la EE (Lizasoain et al., 2016). Una vez controlado el ISEC, la red del centro deja de resultar estadísticamente significativa (Choi y Calero, 2012; Joaristi et al., 2014).

- Sexo del estudiante

El sexo femenino presenta en los resultados de PISA una correlación significativa y negativa con el rendimiento en el área de Matemáticas (Cordero y Manchón, 2014). Los chicos obtienen puntuaciones más altas que las chicas en Matemáticas (8 puntos medios más) y Ciencias. Ellas presentan un nivel más alto en comprensión lectora y lectoescritura, llegando a superar a los chicos en 27 puntos (Gamazo et al., 2018; Ibáñez-Martín y Formichella, 2017; OECD, 2016; Suárez-Enciso et al., 2016). Martínez-Garrido y Murillo (2016b) apuntan que los alumnos obtienen 3 puntos medios más en Matemáticas que las alumnas y estas, a su vez, obtienen 3.5 puntos medios más que ellos en Lectura y Escritura. Ellas obtienen mejores resultados en el área de 
Formación Cívica y Ética y en Lengua, posicionándose 29 y 34 puntos medios por encima de ellos respectivamente (Eggers-Cuellar, 2016).

Un porcentaje superior de alumnas en el centro educativo tiene un efecto positivo y significativo en el rendimiento escolar, aunque no demasiado relevante. Esto sucede debido a que las niñas se desarrollan cognitivamente antes (Merlo-Martínez, 2015).

Sin embargo, en los países más desarrollados, como afirman Lizasoain et al (2016) y Murillo et al (2014), la variable sexo es muy poco significativa, al contrario que en aquellos que se encuentran en vías de desarrollo, donde aún sigue existiendo brecha de sexo (Martínez-Garrido, 2015). Las diferencias de rendimiento entre niños y niñas son tan reducidas que el sexo no se considera una variable relevante en el estudio de la EE (Perry, 2017).

\section{- Asistencia a educación preescolar}

Martínez-Garrido y Murillo (2016c) hallaron que cuando un alumno de nivel socioeconómico familiar bajo es escolarizado en los años anteriores a la educación obligatoria, por lo general, los efectos negativos del ISEC familiar se minimizan. Esto puede deberse a que la escolarización en esta etapa incrementa los resultados en el área de Matemáticas (Cordero y Manchón, 2014; Suárez-Enciso et al., 2016) y, a la postre, en Competencia Lingüística, ya que se estimula el interés de los niños por la lectura (Costa y Araújo, 2018; Kalb y van Ours, 2014; Soodla et al., 2015).

\section{- Asistencia a clase}

El factor asistencia y puntualidad a clase influye directa y positivamente en el rendimiento escolar (lyer y Moore, 2017; Suárez-Enciso et al., 2016). A mayor asistencia del alumnado y profesorado a clase, mejores resultados académicos.

Martínez et al. (2017) defienden que, a nivel alumno, la variable más influyente en el rendimiento de Matemáticas es llegar al aula antes de que el profesor comience la clase, aunque, probablemente, esta sea una correlación espuria explicada por el nivel de interés e implicación del alumnado.

\section{- Participación de las familias en el centro}

Existe una correlación positiva y significativa entre rendimiento académico del alumnado y la implicación de las familias en el centro (Azpillaga-Larrea et al., 2014; Castro-Morera et al., 2014; Castro-Morera et al., 2015; Gomariz-Vicente et al., 2017; Martínez-Garrido, 2015; MartínezGarrido y Murillo, 2016e; Murillo y Krichesky, 2015).

En los centros de baja eficacia, las familias de los alumnos apenas tienen relación con los centros. Esta situación se produce porque las familias tienen niveles educativos muy bajos y/o porque solo se les convoca para hablar del rendimiento de sus hijos o para pedirles participación y colaboración en el mantenimiento del centro (Hernández-Castilla et al., 2014).

Figueroa-Gutiérrez (2017) afirma la existencia de la correlación indicada anteriormente, pero defiende que no es la implicación de las familias lo que favorece un mejor rendimiento y resultados escolares, sino que es el centro eficaz, al aumentar el nivel de rendimiento del alumnado, el que despierta el interés y deseo de participación de las familias.

- Otras variables

El mes de nacimiento del alumnado y los cambios frecuentes de centro son variables que influyen en la EE durante los primeros años de Educación Primaria (Cordero y Manchón, 2014; Gamazo et al., 2018; González-Vallinas et al., 2018; Matta et al., 2016). 


\section{Conclusiones y Discusión}

Atendiendo al objetivo inicial, se han expuesto las variables contextuales más significativas que intervienen en el rendimiento educativo del alumnado. Estas deberían ser consideradas de relevancia en los planes y programas de orientación que dan lugar al ejercicio de la acción tutorial como parte fundamental en los procesos de apoyo educativo. Para llevar a cabo una orientación y tutoría que consiga el desarrollo integral del alumnado y que esté adaptada a sus necesidades, como factor imprescindible en los planes de acción tutorial, debería incluirse el estatus socioeconómico y cultural de las familias. Esta variable es la más estudiada en todas las investigaciones analizadas, siendo la que más influye en el proceso de enseñanza-aprendizaje del alumnado (Murillo et al., 2015).

La participación de las familias en el centro tiene una correlación significativa y positiva con la EE (Ballesteros-Velázquez et al., 2014; Castro-Morera et al., 2015). La formación psicopedagógica del orientador lo convierten en el agente clave para llevar a cabo proyectos relacionados con la orientación familiar (Ramírez-García et al., 2020).

El sexo del estudiante es la variable que más controversia causa entre los autores. Algunos estudios la consideran muy influyente (Cordero y Manchón, 2014; Gamazo et al., 2018; Martínez et al., 2017), otros poco relevante (Lizasoain et al., 2016; Murillo et al., 2014) y un tercer grupo estima que solo afecta a países en vías de desarrollo (Martínez-Garrido, 2015). Esta variable, por tanto, no debería ser obviada. El profesional de orientación educativa es fundamental para articular y garantizar que en los centros se fomente la igualdad a través de actividades y talleres donde se aborde la prevención de la violencia (Decreto 327/2010). Apoyándose en la acción tutorial, el orientador también debe promover acciones educativas que favorezcan la educación en valores y la igualdad de género (Ruiz-García et al., 2020)

La condición de alumnado inmigrante de primera generación resulta un factor negativo para el rendimiento educativo del alumnado (Gamazo et al., 2018; Martínez et al., 2017; OECD, 2016). Otros autores afirman que, una vez controlado el ISEC, esta variable deja de tener relevancia estadística (Choi y Calero, 2012; Joaristi et al., 2014). Aun así, los especialistas de la orientación deben trabajar con la comunidad educativa para potenciar en los centros prácticas inclusivas, reales y adaptadas a su contexto, que verdaderamente generen comportamientos no segregadores y más equitativos (Fernández-Tilve y Malvar-Méndez, 2019; Morales-Carrero, 2020).

La asistencia a la educación preescolar mejora el rendimiento del alumnado (Shafiq et al., 2018). Respecto a la asistencia a clase, las aulas más eficaces se caracterizan por altos niveles de puntualidad, tanto del alumnado como del profesorado (lyer y Moore, 2017). Resulta más que probable que esta sea una correlación espuria determinada por el interés y capacidad del alumnado. No obstante, como actuaciones para el desarrollo global del alumnado, en los programas de orientación se deberían incluir los conceptos de puntualidad y compromiso de cara al futuro de los estudiantes cuando acaben su etapa educativa y salgan al mundo laboral.

El papel motivador de la orientación toma especial relevancia cuando consideramos el rendimiento previo del alumnado. Esta variable guarda una relación positiva y estadísticamente significativa con el rendimiento educativo (Martínez-Garrido y Murillo, 2016b). La acción orientadora debe tener un carácter motivador en el alumnado para que este sea capaz de aumentar su rendimiento educativo con estrategias y técnicas de estudio. 
La orientación psicopedagógica debe atender especialmente el aspecto motivacional en el caso de alumnado repetidor. Esta variable correlaciona negativamente con la EE (Manchón, 2014; Suárez-Enciso et al., 2016). La estrategia de repetición no es efectiva en la mejora de resultados académicos (Choi et al., 2018). Es aquí cuando la orientación debe tener un papel clave en el desarrollo del alumnado, evitando que este se desvincule totalmente de la educación.

Investigaciones como las de Choi y Calero (2012) indican cómo, una vez controlado el efecto del ISEC y la red, los centros privados no son los más eficaces. A los centros públicos asisten familias con ISEC más bajo, que redunda en un nivel de rendimiento más bajo (Merlo-Martínez, 2015; Suárez-Enciso et al., 2016).

A pesar de haber revisado una cantidad considerable de publicaciones de impacto en el periodo 2014-2019, se podría haber ampliado este número de documentos al igual que los años de búsqueda para tener una visión más amplia de lo publicado sobre el tema que se ha abordado. También se podría haber utilizado algún software de análisis de datos cualitativos que permitiera establecer relaciones entre las variables estudiadas. Estas limitaciones nos dan pie para el desarrollo de futuras investigaciones que aborden estos aspectos. Por otro lado, desde la perspectiva de la orientación educativa se podría estudiar cada variable que ha resultado significativa, con el fin de hallar técnicas, estrategias y actividades que desarrollen cada factor contextual relevante en la EE a nivel de alumnado y de centros.

Se concluye, coincidiendo con Ford y Rice (2015), que un mayor control de las variables contextuales, abordables desde el ámbito de la orientación educativa y escolar, contribuye a mejorar los procesos de enseñanza-aprendizaje de los estudiantes. Estos procesos de mejora atendiendo a dichas variables deben apoyarse en el trabajo de los equipos de orientación, desde modelos educativos constructivistas, sistémicos y colaborativos (Oliva et al., 2019), aprovechando la satisfacción que estos muestran en su trabajo con los equipos directivos y tutores en centros de Infantil y Primaria (López y Manzano-Soto, 2019). El centro debe ser un lugar agradable para el alumnado y el profesorado y debe favorecer el proceso de enseñanza-aprendizaje independientemente del contexto de los niños. Debe suavizar las diferencias que puedan existir entre el alumnado, distribuyendo los recursos y oportunidades de forma equitativa (Downey y Condron, 2016).

\section{Referencias bibliográficas}

Abadía, L.K., Bernal, G.L. y Muñoz, S. (2018). Brechas en el desempeño escolar en PISA: ¿Qué explica la diferencia de Colombia con Finlandia y Chile? Archivos Analíticos de Políticas Educativas, 26(82). http://dx.doi.org/10.14507/epaa.26.3423

Aguilera-Eguía, R. (2014). Carta al director. ¿Revisión sistemática, revisión narrativa o metaanálisis? Revista de la Sociedad Española del Dolor, 21(6), 359-360. http://dx.doi.org/10.4321/S1134-80462014000600010

Alm, S., Laftman, S.B., Sandahl, J. y Modin, B. (2019). School effectiveness and students' future orientation: A multilevel analysis of upper secondary schools in Stockholm, Sweden. Journal of Adolescence, 70, 62-73. https://doi.org/10.1016/j.adolescence.2018.11.007 
Amor-Almedina, M.I. y Serrano Rodríguez, R. (2019). Las competencias profesionales del orientador escolar: el rol que representa desde la visión del alumnado. Revista de Investigación Educativa, 38(1), 71-88. https://doi.org/10.6018/rie.321041

Azpillaga-Larrea, V., Intxausti, N. y Joaristi-Olariaga, L. (2014). Implicación de las familias en los centros escolares de alta eficacia en la comunidad autónoma vasca. Bordón, 66(3), 27-37. https://doi.org/10.13042/Bordon.2014.66302

Ballesteros-Velázquez, B., Aguado-Odina, T. y Malik-Liévano, B. (2014). Escuelas para todos: diversidad y educación obligatoria. Revista Electrónica Interuniversitaria de Formación del Profesorado, 17(2), 93-107. https://doi.org/10.6018/reifop.17.2.197351

Baş, G., Şentürk, C. y Ciğerci, F.M. (2017). Homework and academic achievement: A metaanalytic review of research. Issues in Educational Research, 27(1), 31-50. http://www.iier.org.au/iier27/bas.pdf

Ben-Eliyahu, A. y Bernacki, M.L. (2015). Addressing complexities in self-regulated learning: a focus on contextual factors, contingencies, and dynamic relations. Metacognition Learning, 10, 1-13. https://doi.org/10.1007/s11409-015-9134-6

Bisquerra, R. (2012). Metodología de la investigación educativa. La Muralla.

Blanco-Bosco, E. (2008). Factores escolares asociados a los aprendizajes en la educación Primaria mexicana: un análisis multinivel. REICE. Revista Electrónica Iberoamericana sobre Calidad, Eficacia y Cambio en Educación, 6(1), 58-84. https://www.redalyc.org/pdf/551/55160105.pdf

Brandsma, H.P. (1993). Basisschoolkenmerken en de Kwaliteit van Het Onderwijs (Characteristics of primary schools and the quality of education). RION

Brault, M.C., Janosz, M. y Archambault, I. (2014). Effects of school composition and school climate on teacher expectations of students: A multilevel analysis. Teaching and Teacher Education, 44, 148-159. https://doi.org/10.1016/j.tate.2014.08.008

Brookover, W. Beady, C., Flood, P., Schweitzer, J. y Wisenbaker, J. (1979). School social systems and student achievement: schools can make a difference. Praeger.

Castro-Morera, M., Expósito-Casas, E., Lizasoain, L., López, E. y Navarro, E. (2014). Participación familiar y rendimiento académico. Una síntesis meta-analítica. En Ministerio de Educación, Cultura y Deporte, Consejo Escolar del Estado, La participación de las familias en la educación escolar (pp. 83 - 105). Secretaría General Técnica.

Castro-Morera, M., Expósito-Casas, E., López-Martín E., Lizasoain, L., Navarro-Asencio, E. y Gaviria, J.L. (2015). Parental involvement on student academic achievement: A metaanalysis. Educational Research Review, 14, 33-46. https://doi.org/10.1016/i.edurev.2015.01.002

Castro-Morera, M., García-Medina, A., Pedroza-Zúñiga, H. y Caso-Niebla, J. (2015). Escuelas de enseñanza media y valor añadido bajo. Perfiles diferenciales de escuelas secundarias en Baja California. Archivos Analíticos de Políticas Educativas, 23(67). https://doi.org/10.14507/epaa.v23.1917

Choi, A. y Calero, J. (2012). Rendimiento académico y titularidad de centro en España. Profesorado, 16(3), 31-57. https://recyt.fecyt.es/index.php/profesorado/article/view/42385

Choi, A., Gil, M., Mediavilla, M. y Valbuena, J. (2018). Predictors and Effects of Grade Repetition. $\begin{array}{llll}\text { Revista de Economía } & \text { Mundial, } & \text { 21-42. }\end{array}$ http://rabida.uhu.es/dspace/bitstream/handle/10272/14724/Predictors.pdf?sequence=2 
Coleman, J.S., Campbell, E.Q., Hobson, C.J., McPartland, J., Mood, A.M., Weinfeld, F.D. y York, R. (1966). Equality of educational opportunity. Department of Health, Education y Welfare, Office of Education.

Condliffe, B.F., Boyd, M.L. y Deluca, S. (2015). Stuck in school: How social context shapes school choice for inner-city students. Teachers College Record, 117, 1-36. https://krieger.jhu.edu/sociology/wp-content/uploads/sites/28/2012/02/Stuck-in-

School How-School-Choice-Policies-Interact-with-Social-Context-to-Shape-Inner-CityStudents.pdf

Cordero, J.M. y Manchón, C. (2014). Factores explicativos del rendimiento en educación primaria: un análisis a partir de TIMSS 2011. Estudios sobre educación, 27, 9-35. https://doi.org/10.15581/004.27.9-35

Cordero, J., Machón, C. y Simancas, R. (2014). La repetición de curso y sus factores condicionantes en España. Revista de Educación, 365, 12-37. https://doi.org/10.4438/1988-592X-RE-2014-365-263

Costa, P. y Araújo, L. (2018). Skilled Students and Effective Schools: Reading Achievement in Denmark, Sweden, and France. Scandinavian Journal of Educational Research, 62(6), 850-864. https://doi.org/10.1080/00313831.2017.1307274

Danico, M.Y. (2004). Intersections: Asian and Pacific American transcultural studies. University of Hawái Press.

Decreto 327/2010, de 13 de julio, por el que se aprueba el Reglamento Orgánico de los Institutos de Educación Secundaria. Boletín Oficial de la Junta de Andalucía, 139, de 16 de julio de 2010, 8-34. https://www.juntadeandalucia.es/boja/2010/139/boletin.139.pdf

Downey, D.B. y Condron, D.J. (2016). Fifty years since the Coleman report: Rethinking the relationship between schools and inequality. Sociology of Education, 89(3), 207-220. https://doi.org/10.1177/0038040716651676

Edmonds, R. (1979). Effective schools for the urban poor. Educational Leadership, 37(1), 15-24.

Eggers-Cuellar, K. (2016). Factores de eficacia escolar asociados al aprendizaje de alumnos del sistema de Telesecundaria en México [Tesis doctoral, Universidad Complutense de Madrid]. TESEO

Fernández-Fernández, S., Fernández-Alonso, R., Arias-Blanco, J.M., Fernández-Raigoso Castaño, M. y Burguera-Condon, J.L. (2016). Oportunidad de aprendizaje y eficacia docente. Análisis exploratorio de factores asociados. Bordón, 68(4), 49-65. https://doi.org/10.13042/Bordon.2016.38075

Fernández-Tilve, M.D. y Malvar-Méndez, M.L. (2019). Las competencias emocionales de los orientadores escolares desde el paradigma de la educación inclusiva. Revista de Investigación Educativa, 38(1), 239-257. https://doi.org/10.6018/rie.369281

Ferreira-González, I., Urrútia, G. y Alonso-Coello, P. (2011). Revisiones sistemáticas y metaanálisis: bases conceptuales e interpretación. Revista Española de Cardiología, 64(8), 688-696. https://doi.org/10.1016/j.recesp.2011.03.029

Figueroa-Gutiérrez, V.I. (2017). Eficacia escolar en entornos vulnerables de la República dominicana. Estudio de casos múltiples [Tesis doctoral, Universidad Autónoma de Madrid]. Repositorio Institucional. http://hdl.handle.net/10486/679876

Ford, R. y Rice, K. (2015). Value-Added Results for Public Virtual Schools in California. Educational Technology \& Society, 18(4), 412-423. 
Gamazo, A., Martínez-Abad, F., Olmos-Migueláñez, S. y Rodríguez-Conde, M.J. (2018). Evaluación de factores relacionados con la eficacia escolar en PISA 2015. Un análisis multinivel. Revista de educación, 379, 56-84. https://doi.org/10.4438/1988-592X-RE-2017$\underline{379-369}$

Gil-Flores, J. (2014). Factores asociados a la brecha regional del rendimiento español en la evaluación PISA. Revista de Investigación Educativa, 32(2), 393-410. https://doi.org/10.6018/rie.32.2.192441

Gomariz-Vicente M.A., Hernández-Prados, M.A., García-Sanz, M.P. y Parra-Martínez, J. (2017). Tejiendo puentes entre la escuela y la familia. El papel del profesorado. Bordón, 69(2), 415. https://doi.org/10.13042/Bordon.2016.49832

González-Vallinas, P., Librero, J., Peiró, S. y San Fabián, J.L. (2018). Edad relativa y resultados escolares en educación primaria en la Comunidad Autónoma de Cantabria. Archivos Analíticos de Políticas Educativas, 26(141). https://doi.org/10.14507/epaa.26.2926

Gustafsson, J.A. y Hansen, Y.A. (2018). Changes in the Impact of Family Education on Student Educational Achievement in Sweden 1988-2014. Scandinavian Journal of Educational Research, 62(5), 719-736. https://doi.org/10.1080/00313831.2017.1306799

Hanushek, E.A. y Woessmann, L. (2017). Resources and Student Achievement: A Review of Cross-Country Economic Research. In M. Rosen, K. Yang-Hansen y U. Wolff. (Eds.), Cognitive Abilities and Educational Outcomes, Methodology of Educational Measurement and Assessment (149-171). Springer International Publishing. https://doi.org/10.1007/978$\underline{3-319-43473-58}$

Hargreaves, A. (1986). Two cultures of schooling. Falmer Press.

Hendrick, C.E., Cohen, A.K., Deardorff, J. y Cance, J.D. (2016). Biological and socio-cultural factors during the school years predicting women's lifetime educational attainment. Journal of School Health, 86(3): 215-224. https://doi.org/10.1111/josh.12368

Hernández-Castilla, R., Murillo, F.J. y Martínez-Garrido, C. (2014). Factores de ineficacia escolar. REICE. Revista Iberoamericana sobre Calidad, Eficacia y Cambio en Educación, 12(1), 103-118.

Higgins, J.P.T. y Green, S. (Eds.). (2011). Manual Cochrane de revisiones sistemáticas de intervenciones. The Cochrane Collaboration https://es.cochrane.org/sites/es.cochrane.org/files/public/uploads/Manual Cochrane $510 \mathrm{r}$ eduit.pdf

Hill, P.W., Rowe, K.J. y Jones, T. (enero 1995). Factors affecting students' educational progress: Multilevel modelling of educational effectiveness. Congress for School Effectiveness and Improvement, Leeuwarden.

Hofman R.H., Hofman, W.H.A. y Gray, J.M (2015). Three conjectures about school effectiveness: An exploratory study. Cogent Education, 2(1). https://doi.org/10.1080/2331186X.2015.1006977

Hopkins, D., Ainscow, M. y West, M. (1994). School improvement in an era of change, Cassell.

Huang, H-P. y Shih, Y-H. (2017). A study of primary school teachers' attitudes toward teacher valuation for professional development and teaching effectiveness in the remote districts. EURASIA Journal of Mathematics Science and Technology Education, 13(8), 5949-5960. https://doi.org/10.12973/eurasia.2017.01052a 
Ibáñez-Martín, M. y Formichella, M.M. (2017). Logros educativos: ¿Es relevante el sexo de los estudiantes? Archivos Analíticos de Políticas Educativas, 25(3). https://doi.org/10.14507/epaa.25.2520

Ibáñez-Martínez, R. (2020). Origen y evolución de los servicios de orientación en las universidades españolas: hacia un nuevo modelo. Revista de Orientación Educacional, 33(64). http://www.roe.cl/index.php/roe/article/view/95

lyer, P. y Moore, R. (2017). Measuring learning quality in Ethiopia, India, and Vietnam: from primary to secondary school effectiveness. Compare: A Journal of Comparative and International Education, 47(6), 908-924. https://doi.org/10.1080/03057925.2017.1322492

James, M.C., Rupley, W.H., Hall, K.K., Nichols, J.A., Rasinski, T.V. y Harmon, W.C. (2016). Reform stall: An ecological analysis of the efficacy of an urban school reform initiative to improve students' reading and mathematics achievement. Cogent Education, 3(1). https://doi.org/10.1080/2331186X.2016.1245089

Jeynes, W. (2015). A meta-analysis on the factors that best reduce the achievement gap. Education and Urban Society, 47, 523-554. https://doi.org/10.1177/0013124514529155

Joaristi, L., Lizasoain, L. y Azpillaga, V. (2014). Detection and Characterization of Highly Effective Schools in the Autonomous Community of the Basque Country Using Contextualized Cross-Sectional Attainment Models and Hierarchical Linear Models. Estudios sobre Educación, 27, 37-61. https://doi.org/10.15581/004.27.37-61

Kalb, G. y van Ours, J.C. (2014). Reading to young children: A head-start in life? Economics of Education Review, 40,1-24. https://doi.org/10.1016/i.econedurev.2014.01.002

Lizasoain, L. y Angulo, A. (2014). Buenas prácticas de escuelas eficaces del país vasco: metodología y primeros resultados. Participación educativa, 3(4), 17-27. https://doi.org/10.4438/1886-5097-PE

Lizasoain, L., Bereziartua, J.E. y Bartau, I. (2016). La formación permanente del profesorado en centros educativos de alta eficacia. Bordón, 68(2), 199-218. https://doi.org/10.13042/Bordon.2016.68213

López, M.E. y Manzano-Soto, N. (2019). Valoración del nuevo modelo de orientación implementado en la comunidad autónoma de Cantabria por parte de los orientadores educativos. Revista Española de Orientación y Psicopedagogía, 30(2), 108-127. https://doi.org/10.5944/reop.vol.30.num.2.2019.25341

Martínez-Clares, P. (Ed.) (2016). Manual de orientación y acción tutorial. Diego Marín Librero Editor.

Martínez, F., Lizasoain, L., Castro-Morera, M. y Joaristi, L.M. (2017). Selección de escuelas de alta y baja eficacia en Baja California (México). Revista Electrónica de Investigación Educativa, 19(2), 38-53. https://doi.org/10.24320/redie.2017.19.2.960

Martínez-Garrido, C. (2015). Investigación sobre enseñanza eficaz. Un estudio multinivel para Iberoamérica [Tesis doctoral, Universidad Autónoma de Madrid]. TESEO

Martínez-Garrido, C. y Murillo, F.J. (2016a). Investigación iberoamericana Sobre enseñanza eficaz. RMIE. Revista Mexicana de Investigación Educativa, 21(69), 471-499. http://www.scielo.org.mx/scielo.php?script=sci arttext\&pid=S1405$\underline{66662016000200471 \& \operatorname{lng}=\mathrm{es} \& \ln \mathrm{n}=\mathrm{es}}$ 
Martínez-Garrido, C. y Murillo, F.J. (2016b). Incidencia de la distribución del tiempo no lectivo de los docentes en Educación Primaria en el aprendizaje de sus estudiantes. RELIEVE, 22(2). https://doi.org/10.7203/relieve.22.2.9433

Martínez-Garrido, C. y Murillo, F.J. (2016c). Factores de eficacia escolar en la República Dominicana. Innovación Educativa, 16(72). 113-132. http://www.scielo.org.mx/scielo.php?script=sci arttext\&pid=S166526732016000300113\&lng=es\&t|ng=es

Martínez-Garrido, C. y Murillo, F.J. (2016d). Factores de eficacia escolar en Venezuela. Areté. Revista Digital del Doctorado en Educación de la Universidad Central de Venezuela, 2(1), 73-90.

Martínez-Garrido, C. y Murillo, F.J. (2016e). Investigación iberoamericana sobre enseñanza eficaz. RMIE. Revista Mexicana de Investigación Educativa, 21(69), 471-499. http://www.scielo.org.mx/scielo.php?script=sci arttext\&pid=S1405$66662016000200471 \& \operatorname{lng}=$ es\&tlng=es.

Matta, R.B., Ribas, R.P., Sampaio, B. y Sampaio, G.R (2016). The effect of age at school entry on college admission and earnings: a regression-discontinuity approach. Journal of Labor Economics, 5(9). https://doi.org/10.1186/s40172-016-0049-5

McCardle, L. y Hadwin, A.F. (2015). Using multiple, contextualized data sources to measure learners' perceptions of their self-regulated learning. Metacognition Learning, 10, 43-75. https://doi.org/10.1007/s11409-014-9132-0

McEwan, P.J. (2015). Improving Learning in Primary Schools of Developing Countries: A MetaAnalysis of Randomized Experiments. Review of Educational Research, 85(3), 353-394. https://doi.org/10.3102/0034654314553127

Merlo-Martínez, T. (2015). La eficiencia y eficacia de la educación primaria en la comunidad de Madrid [Tesis doctoral, CEU Universidad San Pablo]. TESEO

Morales-Carrero, J.A. (2020). El rol orientador del docente en el contexto comunitario. Revista $\begin{array}{lllll}\text { Española de Orientación y } & \text { Psicopedagogía. } & 31(2),\end{array}$ https://doi.org/10.5944/reop.vol.31.num.2.2020.27984

Mortimore, P. Sammons, P., Stoll, L., Lewis, D. y Ecob, R. (1988). School matters. The junior years. Open Books.

Murillo, F.J. (2003). El movimiento teórico-práctico de mejora de la escuela. Algunas lecciones aprendidas para transformar los centros docentes. REICE. Revista Iberoamericana Sobre Calidad, Eficacia y Cambio en Educación, 1(2). https://revistas.uam.es/reice/article/view/5352

Murillo, F.J. (2005). ¿Importa la escuela? Una estimación de los efectos escolares en España. Tendencias pedagógicas, 10, 29-45.

Murillo, F. J., Hernández-Castilla, R. y Martínez-Garrido, C. (2016). ¿Qué ocurre en las aulas donde los niños y niñas no aprenden? Estudio cualitativo de aulas ineficaces en Iberoamérica. Perfiles Educativos, 38(151), 55-70. https://doi.org/10.22201/iisue.24486167e.2016.151.54886

Murillo, F.J., Hidalgo, N. y Flores, S. (2015). Incidencia del contexto socioeconómico en las concepciones docentes sobre evaluación. Profesorado, Revista de currículum y formación del profesorado, 20(3), 251-281. 
Murillo, F.J. y Krichesky, G.L. (2015). Mejora de la Escuela: Medio siglo de lecciones aprendidas. REICE. Revista Iberoamericana sobre Calidad, Eficacia y Cambio en Educación, 13(1), 69102.

Murillo, F.J., Martínez-Garrido, C. y Hidalgo, N. (2014). Incidencia de la forma de evaluar los docentes de Educación Primaria en el rendimiento de los estudiantes en España. Estudios sobre educación, 27, 91-113. https://doi.org/10.15581/004.27.91-113

Núñez, M. (2007). Las variables: estructura y función en las hipótesis. Investigación Educativa. 11(20), 163-179.

OECD. (2016). PISA 2015 Results (Volume I): Excellence and Equity in Education, PISA. OECD Publishing. https://doi.org/10.1787/9789264266490-en

Oliva, D. de la, Tobón, S., Pérez, A.K., Romero, J. y Escamilla, K.M. (2019). Evaluación del modelo educativo constructivista de orientación educativa e intervención psicopedagógica desde el enfoque socioformativo. Bordón, 55(2), 561-576. https://doi.org/10.5565/rev/educar.772

Olmos-Gómez, M.C., Luque-Suárez, M. y Cuevas-Rincón, J.M. (2020). A Confirmatory Evaluation of an Educational Orientation Tool for Pre-University Students. Education Sciences, 9(4), 285. https://doi.org/10.3390/educsci9040285

Parra, J. D. (2018). Critical realism and school effectiveness research in Colombia: the difference it should make. British Journal of Sociology of Education, 39(1), 107-125. https://doi.org/10.1080/01425692.2017.1330681

Pedroza, H., Peniche, R. y Lizasoain, L. (2018). Criterios para la identificación y selección de escuelas eficaces de nivel medio superior. Revista Electrónica de Investigación Educativa, 20(1), 14-25. https://doi.org/10.24320/redie.2018.20.1.2170

Perry, T. (2017). Inter-method reliability of school effectiveness measures: a comparison of valueadded and regression discontinuity estimates. School Effectiveness and School Improvement, 28(1), 22-38. https://doi.org/10.1080/09243453.2016.1203799

Plowden Committee (1967). Children and their Primary Schools. HMSO.

Prairie, Y.T. y Bird, D.E. (1989). Some misconceptions about the spurious correlation problem in the ecological literature. Oecologia, 81(2), 285-288.

Prendes-Espinosa, M., García-Tudela, P. y Solano-Fernández, I. (2020). Gender equality and ICT in the context of formal education: A systematic review. [Igualdad de género y TIC en contextos educativos formales: Una revisión sistemática]. Comunicar, 63, 9-20. https://doi.org/10.3916/C63-2020-01

Ramberg, J., Låftman, S.B., Almquist, Y. y Modin, B. (2019). School effectiveness and students' perceptions of teacher caring: A multilevel study. Improving Schools, 22(1), 55-7. https://doi.org/ 10.1177/1365480218764693

Ramírez-García, A., Salcines-Talledo, I. y González-Fernández, N. (2020). Los dispositivos móviles en el hogar. Interés formativo de las familias españolas. Revista Española de $\begin{array}{lllll}\text { Orientación } \quad y & \text { Psicopedagogía, } & \text { 3(1), }\end{array}$ https://doi.org/10.5944/reop.vol.31.num.1.2020.27286

Román-Carrasco, M. (2014). Elección y permanencia en escuelas de baja calidad en un sistema educativo de cuasi-mercado. ¿opción o resignación? [Tesis doctoral, Universidad Autónoma de Madrid]. Repositorio Institucional. http://hdl.handle.net/10486/660615 
Ruíz-García, A., Jiménez-Jiménez, O. y Rando-Hurtado, M.A. (2020). Programa de intervención breve de educación sexual desde el modelo biográfico en adolescentes de enseñanza secundaria: un estudio piloto. Revista Española de Orientación y Psicopedagogía, 31(2), 38-55. https://doi.org/10.5944/reop.vol.31.num.2.2020.27985

Rumbaut, R.G. y Portes, A. (2001). Ethnicities. Children of immigrants in America. University of California.

Rutter, M., Mortimore, P., Ouston, J. y Maughan, B. (1979). Fifteen thousand hours. Open Books.

Saminathen, M.G., Låftman, S.B., Almquist, Y.B. y Modin, B. (2018). Effective schools, school segregation, and the link with school achievement. School Effectiveness and School Improvement, 29(3), 464-484. https://doi.org/10.1080/09243453.2018.1470988

Sammons, P., Thomas, S. y Mortimore, P. (1997). Forging links: effective schools and effective departments. Paul Chapman Publishing.

Scheerens, J., Witziers, B. y Steen, R. (2013). A Meta-analysis of School Effectiveness Studies. Revista de Educación, 361, 619-645. https://doi.org/10.4438/1988-592X-RE-2013-361-235

Shafiq, M.N., Devercelli, A. y Valerio, A. (2018). Are there long-term benefits from early childhood education in low- and middle-income countries? Education Policy Analysis Archives, 26(122). https://doi.org/10.14507/epaa.26.3239

Sobrido-Prieto, M. y Rumbo-Prieto, J.M. (2018). La revisión sistemática: pluralidad de enfoques y metodologías. Enfermería clínica. 387-393. https://doi.org/10.1016/i.enfcli.2018.08.008

Soodla, P., Lerkkanen, M.K., Niemi, P., Kikas, E., Silinskas, G. y Nurmi, J. (2015). Does early reading instruction promote the rate of acquisition? A comparison of two transparent orthographies. Learning and Instruction, 38, 14-23. https://doi.org/10.1016/j.learninstruc.2015.02.002

Suárez-Enciso, S., Elías, R. y Zarza, D. (2016). Factores Asociados al Rendimiento Académico de Estudiantes de Paraguay: Un Análisis de los Resultados del TERCE. REICE. Revista Iberoamericana sobre Calidad, Eficacia y Cambio en Educación, 14(4), 113-133. https://doi.org/10.15366/reice2016.14.4.006

Tacconelli, E. (2010). Systematic Reviews. CRD's guidance for undertaking reviews in health care. The Lancet, 10(4). https://doi.org/10.1016/s1473-3099(10)70065-7

Tourón, J., López-González, E., Lizasoain, L., García-San-Pedro, M.J. y Navarro-Asencio, E. (2018). Alumnado español de alto y bajo rendimiento en ciencias en PISA 2015: análisis del impacto de algunas variables de contexto. Revista de Educación, 380, 156-184. https://doi.org/10.4438/1988-592X-RE-2017-380-376

Travitzki, R. (2017). Qualidade com Equidade Escolar: Obstáculos e Desafios na Educação Brasileira. REICE. Revista Iberoamericana sobre Calidad, Eficacia y Cambio en Educación, 15(4), 27-49. https://doi.org/10.15366/reice2017.15.4.002

Urrútia, G. y Bonfill, X. (2010). Declaración PRISMA: Una propuesta para mejorar la publicación de revisiones sistemáticas y metaanálisis. Medicina clínica, 135(11), 507-511. https://doi.org/10.1016/j.medcli.2010.01.015

Weber, G. (1971). Inner-city children can be taught to read: four successful schools. Council for Basic Education. 
Weinstein, J. y Hernández, M. (2016). Birth pains: emerging school leadership policies in eight school systems of Latin America. International Journal of Leadership in Education, 19(3), 241-263. https://doi.org/10.1080/13603124.2015.1020344

Wolhuter, C., Van der Walt, H. y Steyn, H. (2016). A strategy to support educational leaders in developing countries to manage contextual challenges. South African Journal of Education, 36(4), 1-9. https://doi.org/10.15700/saje.v36n4a1297

Fecha de entrada: 6 julio 2020

Fecha de revisión: 6 noviembre 2020

Fecha de aceptación: 23 diciembre 2020 